\title{
Conceptualising Inspiration Networks in Game Design
}

\author{
Xavier Ho, Martin Tomitsch \& Tomasz Bednarz
}

\begin{abstract}
This paper examines the concept of inspiration and proposes a design process for inspiration networks using survey data from Global Game Jam. Global Game Jam is a 48-hour game development competition that invites anyone to participate worldwide. The study illuminates the presence of the famous 'six degrees of separation' effect in these inspiration networks, leveraged to model ideations as related concepts, identifying four major lodestars. It follows research through design, embodying the body of knowledge in the inspiration network as a design artifact. Inspiration networks offer a novel way to discover game genres that have not yet been formally recognised, but have displayed a large influence. The findings offer three design implications: an understanding gained by "thinking with networks", the ability to identify emerging game genres, and the support for intuitive, visual browsing of ideas. Formalisation of inspiration network poses an open field for research.
\end{abstract}

\section{Keywords}

Game Design, Game Culture, Game Development, Game Jam, Ideas, Inspiration Networks, Brainstorming, Visualisation

\section{INTRODUCTION}

Creation of new ideas has been described under many synonyms and related case studies: the 'aha!' moment (Gruber 1981), insightful problem solving (Metcalfe and Wiebe 1987), incubation (Smith and Blankenship 1989), and stroke of insight (Taylor 2006), to list a few. 
Vandenbosch et al. (2001) provide a summary of different theories. It's notable that all theorists agree good ideas could come to us at any moment. Csíkszentmihályi and LeFevre (1989) set out to find the best time and proposed their theory of flow, which suggests we perform at an optimal level when the tasks at hand require a certain degree of skills and provide an ample challenge.

Crites (1971, p. 291) remarked in his article, The Narrative Quality of Experience, "the forms of cultural expression are not historical accidents." The way people speak and the choice of diction are always linked with cultural context, the time and the place from which the language was expressed. In that premise, game development and game culture are no exception to Crites' observation. Genre is the style of expression; mechanisms, the dictionary. Since cultural change is gradual and combinatorial in nature (Popova 2011), ideas are reused, combined, adapted, remixed, twisted, flipped, and perhaps another hundred more ways. Just as modern science builds on existing knowledge and theorems, human creativity, too, builds upon inspiring ideas to fashion.

This article explores game design ideas generated from brainstorming activities. It is not a theoretical piece on why ideas exist (e.g. Ronald et al. 2010), nor how ideas are generated (e.g. Tschang and Szczypula 2006; Hagen 2009, 2010; Kultima and Alha 2011), but what ideas are created, used, and how they are connected by their source of inspiration. We pose the research question, "What are inspiration networks in game design?" and specifically, "What inspiration networks can be found in Global Game Jam?”

The theoretical basis is that there are no truly original game designs. We do not mean that no designs are novel, but states that no designs or design ideas are wholly original, which is to say they must have an origin of inspiration. If an idea is truly original, there would be no familiar ground or indication for communicating the idea, alienating its audience in the process. Hence, an idea must be inspired: there must be an external source of illumination, beyond the self (Thrash and Elliot 2003). In the sense "everything is a remix" (Ferguson 
2011), we see that ideas are reused, combined, connected. We think of ideas as a connected network.

Game development competitions, or game jams, have become popular. A community-driven database shows that at least five game jams are on concurrently worldwide1. Some jams run on a regular basis. For example, Ludum Dare (Latin meaning "to give a game”) runs in April, August and December each year. Global Game Jam, which our study draws its data from, takes place each January. Game jams are usually guided by a central theme. Competing teams have to make games from start to finish within a time frame. One Game A Month events runs every month for the whole month. For Global Game Jam, the time limit is 48 hours.

Due to the short nature of Global Game Jam, games that come out of it are usually simple prototypes with a single focus. This makes them ideal for studying their creators' brainstorming and design processes. However simple games may be, game design is a multidisciplinary craft, including (usually all of) visual art, computer programming, sound design, and gameplay design (Preston et al. 2012, p. 59). In order to work with these skill sets, game jam participants must make quick decisions efficiently and effectively in the game jam. Participants often work in a waterfall fashion (Arya et al. 2013, p. 37), which is to say jammers have little time for design iterations. Ideas are sometimes generated quickly using experimental toolkits with observed learning outcomes (e.g. Kultima et al. 2008; Kultima and Alha 2011).

The evolution of culture is an ever-turning wheel; no human decisions or actions will stop it from turning. Human activities motivate only its direction. We laid out our research interest in the nature of idea evolution and how that can be applied to game studies. In the following section, we will describe the implicit assumption underlined in the connectivity of ideas, the diverse sources of inspiration, and the phenomenal small-world effect (Watts and Strogatz 1998). The section after documents our research 
methodology to examine Global Game Jam design inspiration, and a section following with findings and implications for discussion.

\section{THE IMPLICIT INSPIRATION NETWORK}

In the timeless piece, Music and Moonlight, Sir Arthur O’Shaughnessy (1874, pp. 2-3) captured the concept of inspiration beautifully (revised with modern punctuations):

A breath of our inspiration is the life of each generation: a wondrous thing of our dreaming-unearthly, impossible seeming. The soldier, the king and the peasant are working together in one, till our dream shall become their present, and their work in the world be done.

The definition of inspiration has not changed much for over a century. The English word 'inspiration' originally meant to take in "divine guidance”. It stemmed from Old Latin inspirare, meaning "to breathe in” (Harper 2013). In modern English, getting inspired can mean having a "sudden brilliant or timely idea” (Oxford Dictionaries 2013).

We coin the term inspiration network as a cluster of connected ideas with similar sources of inspiration. A network is a set of nodes with links that connect them. Because networks can be very heavily connected, they are sometimes called complex networks. Complex networks been used to model citation networks (Garfield 1955; Price 1965; Hummon and Doreian 1989; Vazquez 2001; Albert and Barabási 2002, p. 53), blogospheres (Adar and Zhang 2004), design networks (Eckert and Stacey 2000; Grace 2011, p. 19), Wikipedia networks (Schönhofen 2006; Zlatic et al. 2006; Brandes et al. 2009; Massa 2011; Aragon et al. 2012) and births and deaths (Schich et al. 2014), to name a small percentage of the works available.

The concept of an inspiration network can be traced back to Garfield's (1955) citation network. It was first proposed to ease the search of previously published literature and provide a critical system to rank the value of contributions. One decade later, Price (1965) showed that 
citation networks could be used to catalogue journals and classify research papers. Many researchers followed citation networks. Hummon and Doreian (1989) investigated the formation of the DNA theory and identified the critical path of theory coming into existence. They did so by constructing citation networks on 40 of the most cited papers. Adar and Zhang (2004) used hyperlinks in blog posts to construct a network around blogospheres and used their data to derive a ranking system. It is worth noting that half a century since its birth, Garfield's pioneering work in 1955 was reprinted in 2006 in International Journal of Epidemiology with a new title: "A New Dimension in Documentation through Association of Ideas.”

Around that time, online platforms attracted much attention in using networks as a model for research. Wikipedia provided implicit networks of collaborative authorship in different languages (e.g. Zlatić et al. 2006; Brandes et al. 2009), allowing scholars to identify authors' social structures (e.g. Massa 2011; Aragon et al. 2012). These implicit networks are not artificial but naturally occurring (Adar and Zhang 2004). In contrast, LinkedIn's professional networks and Facebook's friendship graphs are explicit networks, connecting profiles in a system. A network snapshot is static, whereas a changing network over a period of time is considered as dynamic or temporal (Schich et al. 2014).

Thrash and Elliot (2003) painted the theory of inspiration with three characteristics: transcendence, motivation, and evocation. Transcendence describes the event in which we see a better vision of what is possible, but does not yet exist. Motivation illustrates the aspiring momentum, a feeling that drives us to do something new. Evocation depicts the action we take as a result of being inspired, and that inspiration must be external, beyond the self. Thrash and Elliot (2004, p. 958) followed up to define the nature of inspiration as a transmission of ideas. There has to be source of inspiration ("being inspired by”) and a destination (“inspire to do”).

The transmission of knowledge is the implicit link in an inspiration network. With a sufficient selection of ideas, we can see a small 
network forming, an evolution of ideas taking place. Through social extensions we may observe the famous 'small-world network' theory, made popular by the film Six Degrees of Separation (Guare 1990; Watts and Strogatz 1998). The theory states that if you know someone who knows someone else, who knows someone else, and so on, the furthest distance of social connections on average is six. Since ideas are constantly being shared between individuals, I argue that inspiration networks must also be part of the small world phenomenon, and the findings should present small clusters of ideas.

An inspiration network is an epistemology model with two elements: an idea (or a manifest thereof) and a link that describes knowledge transmission taking place. Elements can be textual, such as the Global Game Jam survey data used in this study, but inspiration networks can be extended to encompass pictorial, aural and other records. We borrow Vandenbosch et al.'s (2001, p. 109) systematic definition, a "concept or plan formed by mental effort" to describe an idea. In addition, we also consider an idea manifest, such as a game, also as an idea, since the manifestation often embodies the idea itself. They can be physical, lately in more digital and virtual forms. As designer Chimero (2012, p. 50) wrote, a design artifact "is often a physical form, such as a poster, brochure, pottery, painting, or sonnet, but also includes the choices that alter a work's context and placement. Increasingly, these 'artifacts' are becoming less physical, and may take the form of an application, website, or even an experience.”

Metcalfe (2007) explored the construction of connected ideas extensively in his work. In order to aggregate responses from ethnographic data, he organised the data into affinity diagrams, which are groups of notes that share a topic (see Beyer and Holtzblatt 1999) and drew links intuitively between affinity groups to form a problem network. He framed the problem space using that network, noting the 'six degrees of separation' small clusters. He does not specify how he drew the connections, except using a designer's intuition, but remarked the problem network "needs to be about $10-15 \%$ of connections compared to nodes, else the resulting network becomes too cluttered” (2007, p. 146). 
Hagen (2009) examined 25 games published in Sweden in the first decade of the 21st century to find out which parts are "recycled" in these games. He found idea recycling from the same domain (e.g. from games in the same genre or from the genre itself), from narrative and visual art (e.g. films and books), from human activities (e.g. competitive sports or playful events), and from technology and artifacts (e.g. academic research and experimental prototypes). However combined, innovation tends to happen when the source of inspiration is borrowed from a more distant domain than the more dominant one. In some cases, the compounded ideas could turn out to be more effective than the sum of its parts (Tschang and Szczypula 2006).

\section{GLOBAL GAME JAMMING}

Turner et al. (2013, p. 1) praised game jams to have "become an important rite of passage and baptism event, an exploration space and a central indie lifestyle affirmation and community event.” To Turner et al., game designers are artists who create works, or games, whose focus is not about the commercialisation of the outcome, but the process of experiencing the creation of a game in a social context. Other motivations exist as well, including learning a new tool (Fowler et al. 2013a), collaborate in a team environment for self-improvement (Preston et al. 2012), or to be plain, "Getting a chance to make a game with other awesome people in a very short time” (Reng et al. 2013).

Yamane (2013) examined one of Japan’s Global Game Jam site and found the promotion of prototyping was a strong motivation for participants to try it out. The 48-hour duration of game jams fosters quick thinking and decision making, and often the best way to evaluate a decision is to make it and find out. This kind of learning by design practice echoes El-Nasr and Smith's (2006) suggestion that modifying, or modding a game could produce positive learning outcomes. In other words, implemented ideas are favoured over ideas that are merely present on paper.

Game designers often conform their work into an established genre 
in the market, such as a 'first-person shooter' or a 'side-scrolling platformer', and borrow familiar elements from other games, real-life activities, or historical events (Hagen 2009). A study in a previous Global Game Jam found that primary source of inspiration came from the theme, popular gameplay mechanics, other video games, and game genres (Zook and Riedl 2013). Hagen (2012) coined the term lodestars2 to describe the 'designed player experience', the collection of typical mechanics and the desired set of challenges in game design. In Hagen's view, game design can be seen as a combination of recycled ideas, and this process of recycling is to take a group of lodestars into the game design, toss out the ones that don't fit, and bring in new lodestars until the game design is complete.

Many studies on game jams focused on the potential for better learning outcomes. Preston et al. (2012) found that jammers on average have better academic performance than non-jammers, and that attending game jams are perceived as beneficial for improving skills, which also corresponds to Fowler et al.'s (2013a) finding. Reng et al. (2013) described game jams as “an important learning space with characteristics known from communities of practice.” Scholars agree that game jams are a great environment for learning the process of game development as a team.

An inspiration connects in new ideas, and the types of connection of ideas are plentiful. Observed connections from the survey are displayed in Table 1. In this process, it is crucial to have an open mind, to think of idea connections as a diverse event and experience that could come in any flavour, and broadening the possibility of new discovery. To adapt these concepts with a domain, for example, game design, we considered both the type of event and the type of ideas. "Making a voxel sandbox game like Minecraft" could be a 'genre assimilation', for instance, and "porting our shooter game from PC into mobile touch screens” could be 'title translation'. 


\begin{tabular}{|c|c|}
\hline Idea Connection & Example Observation \\
\hline $\begin{array}{l}\text { Similar Origins. Share a } \\
\text { common ancestral origin of } \\
\text { thought. }\end{array}$ & $\begin{array}{l}\text { "Most teams, including mine, ended up boiling the theme } \\
\text { down to the idea of 'perspective'" [P1695]. }\end{array}$ \\
\hline $\begin{array}{l}\text { Assimilation. Belong to } \\
\text { similar schools of thinking and } \\
\text { mental processes }\end{array}$ & $\begin{array}{l}\text { "It's very similar to the game that won [another competition]" } \\
\text { [P99]. }\end{array}$ \\
\hline $\begin{array}{l}\text { Reference. Make a tribute, } \\
\text { mention, or parody of other } \\
\text { ideas. }\end{array}$ & $\begin{array}{l}\text { "Like American McGee's take on Alice, Through the Looking } \\
\text { Glass reinterpreted the tropes of Mario from the perspective of } \\
\text { a drug addict ..." [P1488]. }\end{array}$ \\
\hline $\begin{array}{l}\text { Motivation. Cause a } \\
\text { heightened sense of arousal in } \\
\text { an individual. }\end{array}$ & $\begin{array}{l}\text { "The idea ... started forming naturally along the way, and } \\
\text { before we knew it we were all talking about [the same idea] . } \\
. "[\mathrm{P} 1291] .\end{array}$ \\
\hline $\begin{array}{l}\text { Evocation. Drive someone to } \\
\text { act. }\end{array}$ & "I just built a game I wanted to play" [P603]. \\
\hline $\begin{array}{l}\text { Transcendence. Provide new } \\
\text { insight. }\end{array}$ & $\begin{array}{l}\text { "New idea came up when another member joined that had } \\
\text { much more sense with theme of jam ... " [P83]. }\end{array}$ \\
\hline $\begin{array}{l}\text { Refinement. Move or } \\
\text { frustrate someone to change } \\
\text { something for the better. }\end{array}$ & $\begin{array}{l}\text { "Our scripts collectively threw somewhere around } 800 \\
\text { compiler errors at us ... so we quickly printed out all of our } \\
\text { cards ... and switched to a physical game format" [P1531]. }\end{array}$ \\
\hline $\begin{array}{l}\text { Combination. Compose } \\
\text { several ideas that work well } \\
\text { together. }\end{array}$ & $\begin{array}{l}\text { "We all had different interpretations but eventually agreed } \\
\text { upon an idea that was a combination of several we had come } \\
\text { up with" [P505]. }\end{array}$ \\
\hline $\begin{array}{l}\text { Adaptation. Place into a } \\
\text { different problem domain. }\end{array}$ & $\begin{array}{l}\text { "We tried to come up with an idea involving the theme, but } \\
\text { since none was found, we started crafting a game that was } \\
\text { later adapted to fit the theme" [P1081]. }\end{array}$ \\
\hline $\begin{array}{l}\text { Reuse. Refurbish for a similar } \\
\text { purpose. }\end{array}$ & "[The theme] matched a pre-existing idea" [P144]. \\
\hline $\begin{array}{l}\text { Transmediation. Transform } \\
\text { an idea into another form of } \\
\text { medium. }\end{array}$ & $\begin{array}{l}\text { "It was a game based on [a novel character] and how he saw } \\
\text { things differently than his partner" [P1731]. }\end{array}$ \\
\hline
\end{tabular}

Table 1: List of observed idea connections.

Not only are idea networks qualitative, such as a description of a game and its source of 
inspiration, but they are also quantitative, for example, how many game are similar to it (counting the number of assimilations), and how well received is the title (counting the number of audience reach), if such data is present. By using the numeric relations between ideas, we can begin to see a general understanding of the idea space via the network itself, and interact within it. This interactive understanding helps the design researcher frame the problem space (Schön 1983; Grocott 2011). These qualitative data are implicit to a network, as it depends on the size of the network itself as well as the density of connections, but the trend is immune to the varying windows and size; the more data is input into a network, the more definite the result becomes (Garfield 1955).Constructing Inspiration Networks

Our research aim is to understand what a game design inspiration network is. Our Global Game Jam survey data serves as a corpus. Connected ideas form clusters, hinting at potential trends. We follow a variant methodology of Zimmerman et al.’s (2007) research through design, in combining Schön's (1983) and later Grocott's (2010) reflection-in-action methodology. This separates our research from an ethnographic study; our result is primary the design artifact itself. In brief, the design artifact (the inspiration network) embodies our knowledge of research, and this knowledge can enable other scholars in their research. More importantly, our design process can be applied and repeated as a design methodology in future studies.

After Global Game Jam 2014 came to completion, a survey was administered by the Global Game Jam Research Committee. We proposed to analyse a portion of the survey, which aligns with our research aims in understanding the ideation process at game jams. We obtained university ethics approval and received data from the Global Game Jam Research Committee for analysis. Each individual record was identified by numeric identifiers and could not be traced back to the original respondent. A second numeric identifier was provided to link respondents together from the same participating team.

Games that come out of Global Game Jam are small in design scope and often focused in a single type of gameplay. Participating jammers 
are given a central theme to incorporate into their games, this year's theme being "We don't see them as they are, we see them as we are”. Global Game Jam 2014 gathered 23,198 game developers across 488 jam sites from 72 countries to make a game in 48 hours, with 4,290 games submitted. The relative simplicity in game design and the size of the competition makes an ideal source to study an idea network for a time-constrained event. At the end of the jam, a 60-question survey was sent out to all the participants, and 1,815 respondents were recorded.3

According to the survey report, the average Global Game Jam 2014 participant was 25 years old with a bachelor's degree and has published between 2 to 3 games in the past. $72 \%$ of the teams were formed at least partly before the jam, and 67\% of the respondents developed new connections and worked with someone they did not know before. While the average participant reported the theme was only "somewhat" inspiring, they also reported general satisfaction ( 3.9 out of $5, n=1,671$ ), enjoyed their game jam experience ( 4.3 out of $5, n=1,689)$ and had a lot of fun (4.5 out of 5, $n=1,681)$. Participants strongly indicated that they would participate again next year (4.6 out of $5, n=1,684$ ). Not every question required an answer, hence the difference in the total number of recorded data.

We employed a mixed method to analyse our data set $(n=1,815)$ and separated qualitative and quantitative questions. Not all questions were required. Respondents rated the theme "somewhat" inspiring (3.1 out of $5, n=1,346$ ), even though they considered the theme "a lot" (3.8 out of $5, n=1,343)$. Qualitative data $(n=1,356)$ were free-response answers to open-ended questions regarding their brainstorming process, how they used their theme, and if they changed their idea halfway through the jam.

The theme for Global Game Jam 2014 was “we don’t see things as they are, we see things as we are.” Jammers worked in groups and brainstormed with time constraints in mind. In our findings, one respondent recalled vividly about their brainstorming session: "The theme ... made us think about perception and opposing world views. 
Early on in our design discussions we agreed that we wanted the core mechanics to express some sort of idea related to these ideas. Through word association, we brainstormed a lot of different possible thematic directions for our game, then narrowed those down based on design risk, implementation risk, and personal [preferences and interests]" [P1585].

Most teams worked the theme into their game design, but many of them expressed that the theme was "somewhat vague" and "offered no clear restriction". We hypothesise this was because themes from previous years were more concrete; for example, in 2011, "Extinction" was the theme and in 2013, "Sound of a heartbeat". This year's theme was relatively more abstract. For the most part, the theme was considered during the game design stage. One respondent summarised how the theme was used: "The theme seemed pretty vague, but it did trigger the initial idea for our game. In the end, the theme was neither a huge help or a huge hindrance. It certainly wasn't essential to our game, but it was certainly identifiable in our game" [P131].

Some participants used brainstorming techniques such as word association and mind mapping, both of which generate idea networks. Ideas were written down freely at the start, and the best ones are decided usually by voting. One respondent recorded, "We mixed [our ideas] together and had an anonymous no-judgement voting on ideas. The top three ideas were re-voted upon to determine the best idea for the theme" [P1021].

Gradually, ideas that were implemented would be favoured over the ones that aren't. Jammers were pressed for time, and so if an idea didn't work in implementation, it would be quickly scraped and be replaced by another one. One respondent recalled, "We started by brainstorming what the theme even meant. Then we used a thoughtmap to narrow down the ideas. We then did quick tech prototypes of our top ideas to see which was viable, before making a final decision” [P1092]. 
The free-response data was encoded by tagging phrases which signify or represent an idea, for example, a game design idea, a thought process that could generate ideas, or an object that could inspire other ideas, as outlined earlier. The tags were in nature the type of idea itself, for example, "brainstorming”, “change of mind”, and "frustrations". After a sizeable set of tags was created, we began to construct an affinity diagram (Beyer and Holtzblatt 1999) and grouped similar ideas together. We limited ourselves to a two-tier affinity diagram to preserve the balance between generalisation and details. When a response contained multiple key phrases that signified different ideas or processes, we treated them as separate ideas in our affinity diagram. While this lengthened the research process, it ensured each idea stood by itself.

In the meantime, the authors manually performed keyword extraction for each record. We later used these keywords to construct the links between ideas, so we chose to identify subcategories that are more concrete. For instance, one response was "In our game, you can transform between a child and an adult. The child sees the world in a certain way, the adult sees the world in another way" [P48]. The main idea here was 'transformation', and the subcategories were 'adult' and 'child'. To prevent idea boundaries from becoming too blurry to be of any value, we merged identical (or at least sufficiently similar) ideas into one, saving ourselves from more work later. For instance, we considered 'transformation' and 'change perspective' as the same category, and there were many games which employed that idea.

After the encoding was complete with an affinity diagram, we started by picking one column and place it on the canvas. We chose to place the diagram in a circular fashion and only employ arcs, similar to that of Lombardi's diagrams (2000). To construct the connections, we placed ideas that share the same tag closer to each other, and drew a line between two ideas that shared the same subcategory. This process was repeated until the column was exhausted, and we picked a relatively similar column and repeated the process until the affinity diagram was exhausted. To keep the inspiration network on topic, we excluded records that weren't directly related to ideation 
or brainstorming. For example, "technical issues" was one of the omitted columns. Figure 1 shows our result using a subset of our dataset $(n=178)$, a sufficient number of samples such that small networks could be observed.

\section{VISUALISING INSPIRATION AS A NETWORK}

The human eye is observant in recognising patterns and focal points. For instance, if we were to examine the most parodied games in the mobile space of 2014, we might be able to pick up popular game titles upon visual inspection of a network, and understand the dense and sparse areas of the data space (Auber et al. 2003). A visual representation of a network has an effect on human audience and induce appreciation (Judelman 2004), provides hints and reference (Holmquist and Skog 2003; Manovich 2011) and it supports the process of data analysis (Keim 2002; Stasko et al. 2008). The aesthetics of the network drawing could aid in understanding of sequential events (Twidale and Nichols 1996), reinforce memorability (Borkin et al. 2013), and uplift the potential for interdisciplinary works (Samsel 2013). To make the process more accessible to researchers and analysts, many have continued to maintain an initiative in the area of network visualisation software (e.g. De Nooy et al. 2005; Reas and Fry 2007; Viegas et al. 2007; Bastian et al. 2009; Bostock et al. 2011), which has fostered a rich field of visualisation research and encouraged many interested individuals to explore datasets. 


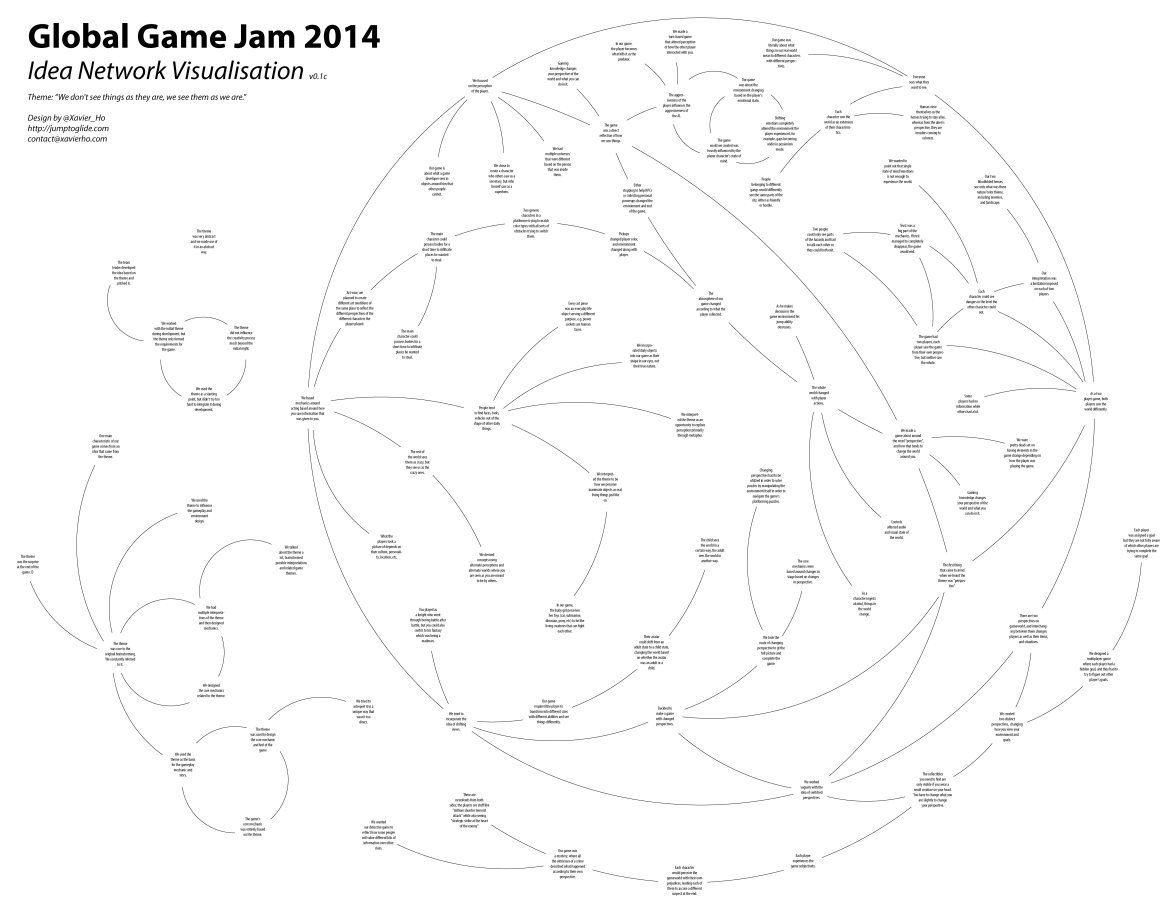

Figure 1: Global Game Jam 2014 Inspiration Network Visualisation4 (full resolution included in endnotes).

Visualisation created from inspiration networks have also been explored as an object of art. Lombardi (2000) in particular expressed the events of financial exchanges and the entities involved as curvature drawings of network graphs by hand. His drawing style often followed a series of aesthetic arcs and expressed a strong sense of direction by the dynamics of lineflow, and they are an inspiration to our network visualisation. Many scholars have worked on algorithms in attempt to reproduce its aesthetics (e.g. Duncan et al. 2010; Chernobelskiy et al. 2012; Eppstein 2013). This paper is not an algorithmic instruction to computerise the act of drawing, but to conceptualise the elements of drawing and exemplify the meanings of elements within the drawing itself.

To effectively design a visual drawing of an inspiration network, we must first find a visual metaphor appropriate for the message. Rather than a generic drawing, a visual metaphor "in aesthetic perception and 
its objects is more conspicuous, salient” (Aldrich 1968, 75). Datasets may have natural metaphors that are inherently visual or sequential, for example, geographic and time (e.g. Raper 2012; Schich et al. 2014) and flight numbers (e.g. Krebs 2002). The style of visualisation also has an impact on the level of insight that could be observed (Vande Moere et al. 2012). At any rate, inspiration networks do not have a direct mapping to any intuitive spatial representations. The challenge, then, is the quest to define a visual metaphor suitable for the data space, optimised for understanding and free exploration.

One major principle for the ease of exploration is a planar drawing, that is to say, the lines do not cross each other unless the intersection is a connection (Masucci et al. 2009). Good examples surround our daily lives; for example, maps of metro public transportation would be terrible if the train lines had crossings that were not an interchange, incorrectly presenting travel information and causing great inconvenience (Hong et al. 2006). For the graph to be obvious at first glance, it must follow that principle.

Secondly, graphs that contain too many nodes would look too crowded, overloading the audience with indistinguishable information. This may be used to display density, such as using transparency and overlaying multiple transparent layers to emphasise heavier areas of data (Vande Moere et al. 2012), but as far as network visualisations go, they lead to too much clutter. Fortunately, inspiration networks, like many others, are likely to display features of small-world networks (Watts and Strogatz 1998), as clusters of grouped, inter-connected nodes would form. Auber et al. (2003) leveraged this characteristic and proposed to reduce information by re-representing a cluster of nodes as one parent node, in effect generating a hierarchical display, progressively revealing information based on the semantic zoom (Auber et al. 2003). Google Maps implements an excellent example of progressive display. After all, we live "in an age of information overload" (Roland and Bawden 2012, p. 226). 


\section{FINDING INSIGHT FROM INSPIRATION NETWORK}

Using the inspiration network in Figure 1, similar ideas are identifiable by the 'islands' they visually form. Some ideas are more or less a unique outlier and have only a couple of connections. One idea was a lone wolf: "The theme was very abstract and we made use of it in an abstract way" [P66]. Reflecting the "somewhat" rating of the theme, some respondents reported the theme "did not influence the creativity process much beyond the initial night” [P40] and they "didn't try too hard to integrate [the theme] during development" [P84]. Contrasting that group, there is another cluster of responses which reported positively about using the theme. These jammers not only "brainstormed possible interpretations and related game themes" [P21] but also "used the theme to influence the gameplay and environment design" [P185]. One team said "the theme was the surprise at the end of the game :D” [P206].

From the islands we found many jammers played with the idea of 'player interpretations'. Many teams took the theme in developing the art direction for their games. One team created "different art renditions of the same place to reflect the different perspectives of the different characters" [P217], while another team focused on the perception of the player, deriving "concepts using alternate perceptions and alternate worlds where you are seen as you are meant to be by others" [P120]. It is interesting to note that some jammers reported "people tend to find faces, tools, vehicles out of the shape of other daily things" [P76], for example, perceiving "inanimate objects as real, living things just like us . . . like a baby girl who perceives her toys (car, submarine, dinosaur, pony, etc) to be like living creatures that can fight each other" [P196], finding "power sockets [which look like] human faces” [P209], in effect, they allowed the players to “explore perception primarily through metaphor” [P46].

The gameplay from 'changing perspectives' and 'transformation' was described by one respondent to be "the most obvious path" in making the games [P164]. To start, one jamming team "required the player to transform into different sizes with different abilities and see things 
differently" [P175]; another game allowed the player to "shift from an adult state to a child state, changing the world based on whether the avatar was an adult or a child” [P218]. This type of game design forced the player to "change what [the characters] are ... to change your perspective" [P85]. In order to proceed further into the game, the player must take on another perspective or role, switching between game avatars.

When jammers considered cooperative or multiplayer options, the theme inspired the concept of 'hidden information'. When the game reveals different information to each player, it introduces dynamic and conflict, or the need to trust each other. One respondent recalled, "Trust was a big part of the mechanics. If trust managed to completely disappear, the game would end," because in the game "each character could see dangers in the level the other character could not” [P81]. Another team with a similar idea designed their game levels such that "two people could only see parts of the hazards and had to talk each other so they could both exit." They also recollected experiencing great humour when one player deliberately lied to the other, causing their avatars to die [P115].

There were also games which changed based on the players' actions, for which we call them 'reactive games' here, for example, in one game, "the player becomes what kills it as the predator" [P204], allowing the losing party to gain some upper hand by taking a comparatively stronger form with a chance for revenge. The games may also shift depending on the aggressiveness of the player, for instance, when a player acts nicely, the non-player characters and the environment also acts friendly and serene; however, if the player chooses to act aggressively throughout the game, they will encounter similar reactions during the game. The actions could either be "stopping to help NPCs or collecting personal powerups," which “changed the environment and end of the game" [P147].

\section{DISCUSSION ON LIMITATIONS AND DESIGN IMPLICATIONS}


We note three design implications learned from game design inspiration networks. The first is an understanding gained by "thinking with networks". The design process to construct an inspiration network is a traversal of networks in nature, which assists our understanding in the connectivity of ideas. In our experience, it was more efficient to start drawing with the larger clusters, as they will provide a healthy number of connections at the start. The designer has more than just the inspiration network as well; we gained insight from the initial process of constructing the affinity diagram, providing us a high-level overview and a new perspective. By identifying subcategories of ideas, we had a second, more implicit layer of understanding on the domain of game design ideas. It is this process of reflection-in-action that illuminates insight in our research (Schön 1983; Grocott 2010). The inspiration network which embodies the connection of ideas can also be distributed to others, transmitting understanding visually.

From the inspiration network we observe the small-world effect. The visual drawing suggests ideas are indeed inter-connected. Since inspiration networks have connectivity, the second design implication offers an ability to identify emerging game genres, such as 'player's interpretation', 'changing perspective', 'transformation', 'reactive games' found in the dataset. The inspiration network allows us to examine the existing relationship between ideas and idea formations, telling a visual story of the construction of ideas. However, as ideas become many, the visualisation becomes too small to read given a fixed-size display, for example, a 24-inch computer monitor. On the other hand, When printed as contemporary or information art in a physical environment, audiences will read a picture up-close when they are presented as tall as themselves (Rav-Acha et al. 2005), which also may inspire curiosity. Digitally, we could support the action of 'zooming' by attaching an interaction with the mouse scroll to see up close, or implement multi-scale visualisations (Auber et al. 2003).

The third design implication is that inspiration networks support intuitive, visual browsing of ideas. The visualisation allowed us to examine the body of knowledge intuitively, retrieving and comparing 
ideas at ease to formulate a formal understanding. If our data set was linked with the games themselves, we have a two-dimensional graph: one in idea-idea connectivity and one in idea-game connectivity. This would enable an audience to browse existing Global Game Jam games not only by title, genre, country, keywords, but also the similarity in idea, design, process, and output. The potential for this application is a future work of our research roadmap.

This study is limited to the scope of Global Game Jam 2014, and with a survey participation rate at only around $7.5 \%$ of the total participants $(1,815$ out of 23,198$)$, our data could not fully represent the population. While the image of the entire network could be extremely interesting to some, we feel the portions which we have compiled into an inspiration network provide ample room for inspection. Secondly, the survey was administered in English, and naturally it may have excluded many jammers from the ability to reply. In the future, perhaps an international study comparing different cultures speaking different languages could be of interest. Because the dataset is only compiled from a survey, the result can only be derived from the jammers' self-reflection. There is therefore an opportunity to conduct an ethnographic study on-site. We also do not know how the ideas evolved throughout Global Game Jam, because most participants only responded to how their final product used the theme; few noted how the ideas were changed.

\section{CONCLUSION}

We proposed an array of contextualised idea connections from Global Game Jam participants, and examined them for common sources of inspiration. Our design process generated a graph drawing that we coin an inspiration network, which supported our investigation in understanding the relations of ideas. The design of an inspiration network suggests that the small-world effect does indeed exist in it, and the visualisation allows us to retrieve insights relatively quickly, given the understanding gained from constructing the network itself. Global Game Jam has been a great opportunity to investigate the ideation and brainstorming processes in game design, and thanks 
to the 48-hour time constraints, the ideas developed were relatively simple and well-focused. Our findings also suggest that given both idea-idea relations and idea-game relations, one can find similar games with related sources of inspiration. Future work of our research includes formalising the drawing and design process of an inspiration network, employing a computer-assisted program to quicken the network generation process, investigating the properties of an inspiration network and what other insights we may extract from it through user studies, and the application for historical exploration.

\section{ACKNOWLEDGEMENTS}

We would like to express our gratitude to the Global Game Jam 2014 Research Committee and for the continuous support from Assistant Professor Jay O'Toole, Georgia State University. We also wish to say thank you to the Global Game Jam 2014 participants and site organisers.

\section{Endnotes}

1. The most up-to-date database is probably CompoHub (http://compohub.net/), a community-maintained website that hosts a timeline graph of what is on the calendar.

2. Previously at DiGRA Australia, our submission used an incorrect term, 'lodestone'. The authors regret making that error and have made the amendment in this paper.

3. These data were derived by the Global Game Jam from a survey conducted following the 2014 Global Game Jam. The Global Game Jam specifically disclaims responsibility for any analyses, interpretations, or conclusions.

The full resolution of Figure 1 is available via this link: https:/github.com/Spaxe/papers/blob/master/ 
Conceptualising\%20Inspiration\%20Networks\%20in\%20Game\%20Design/ Figure-1-print.png

\section{Bibliography}

Adar, E. and L. Zhang. "Implicit Structure and the Dynamics of Blogspace." In 13th Workshop on the Weblogging Ecosystem, 16989-95, 2004.

Albert, R. and A-L Barabási. "Statistical Mechanics of Complex Networks.” Reviews of Modern Physics vol. 74, no. 1(2002): 47-97. doi:10.1103/RevModPhys.74.47.

Aldrich, V. C. “Visual Metaphor.” Journal of Aesthetic Education vol. 2, no. 1(1968): 73-86.

Aragon, P., D. Laniado, A. Kaltenbrunner, and Y. Volkovich. "Biographical Social Networks on Wikipedia." In Proceedings of the Eighth Annual International Symposium on Wikis and Open Collaboration. New York, New York, USA: ACM Press, 2012. doi:10.1145/2462932.2462958.

Arya, A., J. Chastine, J. Preston, and A. Fowler. “An International Study on Learning and Process Choices in the Global Game Jam.” International Journal of Game-Based Learning vol. 3, no. 4(2013): 27-46. doi:10.4018/ijgbl.2013100103.

Auber, D., Y. Chiricota, F. Jourdan, and G. Melançon. "Multiscale Visualization of Small World Networks.” In Proceedings of the Ninth Annual IEEE Conference on Information Visualization, 75-81, 2003.

Bastian, M., S. Heymann, and M. Jacomy. "Gephi: An Open Source Software for Exploring and Manipulating Networks.” ICWSM vol. 8, (2009): 361-62.

Beyer, H, and K Holtzblatt. “Contextual Design.” Interactions vol. 6, no. 1(1999): 32-42. 
Borkin, M. A., A.A. Vo, Z. Bylinskii, P. Isola, S. Sunkavalli, A. Oliva, and H. Pfister. "What Makes a Visualization Memorable?" IEEE Transactions on Visualization and Computer Graphics vol. 19, no. 12(2013): 2306-15. doi:10.1109/TVCG.2013.234.

Bostock, M., V. Ogievetsky, and J. Heer. "D3: Data-Driven Documents." IEEE Transactions on Visualization and Computer Graphics vol. 17, no. 12(2011): 2301-9. doi:10.1109/ TVCG.2011.185.

Brandes, U., P. Kenis, J. Lerner, and D. van Raaij. "Network Analysis of Collaboration Structure in Wikipedia.” In Proceedings of the 18th International Conference on World Wide Web. New York, New York, USA: ACM Press, 2009. doi:10.1145/1526709.1526808.

Chernobelskiy, R., K. Cunningham, M. T. Goodrich, S. G. Kobourov, and L. Trott. "Force-Directed Lombardi-Style Graph Drawing." Graph Drawing, January(2012): 320-31.

Chimero, F. The Shape of Design. Edited by Mandy Brown. Shapco Printing, 2012.

Crites, S. "The Narrative Quality of Experience." Journal of the American Academy of Religion vol. 39, no. 3(1971): 291-311.

Csíkszentmihályi, M., and J. LeFevre. “Optimal Experience in Work and Leisure.” Journal of Personality and Social Psychology vol. 56, no. 5(1989): 815-22.

de Nooy, W., A. Mrvar, and V. Batagelj. Exploratory Network Analysis with Pajek. Cambridge University Press, 2005.

de Solla Price, D. "Networks of Scientific Papers.” Science vol. 149, no. 3683(1965): 510-15.

Duncan, C., D. Eppstein, M. Goodrich, S. Kobourov, and M. Nöllenburg. "Lombardi Drawings of Graphs." Journal of Graph Algorithms and Applications vol. 16, no. 1(2010): 85-108. 
Eckert, C., and M. Stacey. "Sources of Inspiration: A Language of Design.” Design Studies vol. 21, no. 5(2000): 523-38.

El-Nasr, M-S., and B. Smith. "Learning through Game Modding." Computers in Entertainment vol. 4, no. 1(2006): 7. doi:10.1145/ 1111293.1111301.

Eppstein, D. "Planar Lombardi Drawings for Subcubic Graphs." Graph Drawing, January, 2013, 126-37.

Ferguson, K. "Everything Is a Remix.” Vimeo Staff Picks, 2011. https://vimeo.com/14912890

Fowler, A., F. Khosmood, A. Arya, and G. Lai. "The Global Game Jam for Teaching and Learning." In Proceedings of 4th Annual Conference of Computing and Information Technology Research and Education New Zealand, Edited by Michael Verhaart and Mike Lopez. Hamilton, New Zealand, 2013a.

Fowler, A., F. Khosmood, and A. Arya. "The Evolution and Significance of the Global Game Jam.” In Workshop Proceedings of the 8th International Conference on the Foundations of Digital Games, 2013b.

Garfield, E. “Citation Indexes for Science.” Science vol. 122, no. 3159(1955): 108-11.

Garfield, E. "Citation Indexes For Science. A New Dimension in Documentation through Association of Ideas.” International Journal of Epidemiology vol. 35, no. 5(2006): 1123-27. doi:10.1093/ije/ dyl189.

Global Game Jam. “GGJ 2014 by the Numbers,” 2014. http://globalgamejam.org/news/ggj-2014-numbers

Global Game Jam. "Findings From the Post GGJ Survey,” 2014. https://docs.google.com/file/d/0B40cfo58q7S8d0xsRndpZ0xYYUU/ edit 
Grace, K. "Interpretation-Driven Association in Design." The University of Sydney, 2011.

Grocott, L. "Design Research \& Reflective Practice: The Facility of Design-Oriented Research to Translate Practitioner Insights into New Understandings of Design.” RMIT University, 2010.

Gruber, H. "On the Relation Between 'Aha Experiences' and the Construction of Ideas.” History of Science vol. 19, March(1981): 41-59.

Guare, J. “Six Degrees of Separation: A Play.” USA: Random House LLC, 1990.

Hagen, U. "Where Do Game Design Ideas Come From? Innovation and Recycling in Games Developed in Sweden.” In Breaking New Ground: Innovation in Games, Play, Practice and Theory. Brunel University, 2009.

Hagen, U. "Designing for Player Experience: How Professional Game Developers Communicate Design Visions.” In Proceedings of the 2010 International DiGRA Nordic Conference, 2010.

Hagen, U. "Lodestars for Player Experience: Ideation in Videogame Design”. Stockholm University, 2012.

Harper, D. “Inspiration.” Online Etymology Dictionary, 2013. Accessed 10 September, 2013. http://www.etymonline.com/ index.php?term=inspiration

Holmquist, L. E., and T. Skog. "Informative Art: Information Visualization in Everyday Environments.” In Proceedings of the 1st International Conference on Computer Graphics and Interactive Techniques in Australasia and South East Asia - GRAPHITE '03. New York, New York, USA: ACM Press, 2003. doi:10.1145/ 604471.604516.

Hong, S-H., D. Merrick, and H. do Nascimento. “Automatic 
Visualisation of Metro Maps.” Journal of Visual Languages \& Computing vol. 17, no. 3(2006): 203-24.

Hummon, N., and P. Doreian. "Connectivity in a Citation Network: The Development of DNA Theory.” Social Networks vol. 11, (1989): 39-63.

Kaufman, J. and R. Beghetto. "Beyond Big and Little: The Four c Model of Creativity." Review of General Psychology vol. 13, no. 1(2009): 1-12. doi:10.1037/a0013688.

Keim, D. "Information Visualization and Visual Data Mining.” IEEE Transactions on Visualization and Computer Graphics vol. 8, no. 1(2002): 1-8. doi:10.1109/2945.981847.

Krebs, Valdis. "Mapping Networks of Terrorist Cells.” Connections vol. 24, no. 3(2002): 43-52.

Kultima, A., and K. Alha. "Using the VNA Ideation Game at Global Game Jam.” In Proceedings of DiGRA 2011 Conference: Think Design Play. DiGRA/Utrecht School of the Arts, 2011.

Kultima, A., J. Niemelä, J. Paavilainen, and H. Saarenpää. "User Experiences of Game Idea Generation Games.” In Proceedings of Meaningful Play Conference. East Lansing, MI, USA, 2008.

Judelman, G. “Aesthetics and Inspiration for Visualization Design: Bridging the Gap Between Art and Science." In Proceedings to Eighth International Conference on Information Visualisation, 245-50. IEEE, 2004. doi:10.1109/IV.2004.1320152.

Lombardi, M. “BCCI-ICIC \& FAB”. New York: Whitney Museum of American Art, 2000.

Massa, P. "Social Networks of Wikipedia." In Proceedings of the 22nd ACM Conference on Hypertext and Hypermedia. New York, New York, USA: ACM Press, 2011. doi:10.1145/1995966.1995996. 
Masucci, A. P., D. Smith, A. Crooks, and M. Batty. "Random Planar Graphs and the London Street Network." The European Physical Journal B-Condensed Matter and Complex Systems vol. 71, no. 2(2009): 259-71. doi:10.1140/epjb/e2009-00290-4.

Manovich, . "What Is Visualisation?” Visual Studies vol. 26, no. 1(2011): 36-49. doi:10.1080/1472586X.2011.548488.

Metcalfe, M. "Problem Conceptualisation Using Idea Networks." Systemic Practice and Action Research vol. 20, no. 2(2007): 141-50. doi:10.1007/s11213-006-9056-y.

Metcalfe, J, and D Wiebe. "Intuition in Insight and Noninsight Problem Solving.” Memory \& Cognition vol. 15, no. 3(1987): 238-46.

O’Shaughnessy, A. Music and Moonlight. The Metropolitan Magazine, 1874.

Oxford Dictionaries. “'Inspiration.”” Oxford University Press, 2013.

Popova, M. "Networked Knowledge and Combinatorial Creativity.” Brain Pickings, 2011. http://www.brainpickings.org/index.php/2011/ 08/01/networked-knowledge-combinatorial-creativity/

Preston, J., J. Chastine, C. O’Donnell, T. Tseng, and B. MacIntyre. “Game Jams.” International Journal of Game-Based Learning vol. 2, no. 3(2012): 51-70. doi:10.4018/ijgbl.2012070104.

Raper, S. “Graphing the History of Philosophy,” 2012. Accessed 25 November, 2013. http://drunks-and-lampposts.com/2012/06/13/ graphing-the-history-of-philosophy/

Rav-Acha, A., Y. Pritch, D. Lischinski, and S. Peleg. “Dynamosaics: Video Mosaics with Non-Chronological Time.” In 2005 IEEE Computer Society Conference on Computer Vision and Pattern Recognition (CVPR'05), 58-65. IEEE, 2005. doi:10.1109/ CVPR.2005.137. 
Reas, C., and Fry, B. Processing: A Programming Handbook for Visual Designers and Artists. Cambridge, Massachusetts: MIT Press, 2007.

Reng, L., H. Schoenau-Fog, and L. Kofoed. "The Motivational Power of Game Communities-Engaged through Game Jamming." In Proceedings of the 8th International Conference on the Foundations of Digital Games: Society for the Advancement of the Science of Digital Games. Foundations of Digital Games, 2013.

Roland, L., and D. Bawden. "The Future of History: Investigating the Preservation of Information in the Digital Age.” Library \& Information History vol. 28, no. 3(2012): 220-36. doi:10.1179/ 1758348912Z.00000000017.

Ronald, A. K., R. A. Beghetto, and M. A. Runco. "Theories of Creativity." Journal of Biocommunication vol. 17, (2010):20-47.

Ryan, R., and E. Deci. "Self-Determination Theory and the Facilitation of Intrinsic Motivation, Social Development, and WellBeing.” American Psychologist vol. 55, no. 1(2000): 68-78. doi:10.1037//0003-066X.55.1.68.

Samsel, F. "Art-Science-Visualization Collaborations; Examining the Spectrum.” In Proceedings of the IEEE VIS Arts Program (VISAP), 2013.

Schich, M., C. Song, Y-Y. Ahn, A. Mirsky, M. Martino, A-L. Barabasi, and D. Helbing. "A Network Framework of Cultural History.” Science vol. 345, no. 6196(2014): 558-62. doi:10.1126/ science.1240064.

Schön, D. The Reflective Practitioner: How Professionals Think In Action. Basic Books, 1983.

Schönhofen, P. "Identifying Document Topics Using the Wikipedia Category Network.” In 2006 IEEE/WIC/ACM International 
Conference on Web Intelligence, 456-62. IEEE, 2006. doi:10.1109/ WI.2006.92.

Smith, Steven M., and Steven Blankenship. "Incubation Effects." Bulletin of the Psychonomic Society vol. 27, no. 4(1989): 311-14.

Stasko, J., C. Görg, and R. Spence. “Jigsaw: Supporting Investigative Analysis Through Interactive Visualization.” Information Visualization vol. 7, no. 2(2008): 118-32. doi:10.1057/ palgrave.ivs.9500180.

Taylor, J. B. My Stroke of Insight: A Brain Scientist's Personal Journey, 2006. doi:10.1080/13576501003621194

Thrash, T. and A. Elliot. "Inspiration as a Psychological Construct." Journal of Personality and Social Psychology vol. 84, no. 4(2003): 871-89. doi:10.1177/097133360101300201.

Thrash, T. and A. Elliot. "Inspiration: Core Characteristics, Component Processes, Antecedents, and Function.” Journal of Personality and Social Psychology vol. 87, no. 6(2004): 957-73. doi:10.1037/0022-3514.87.6.957.

Tschang, T. and J. Szczypula. "Idea Creation, Constructivism and Evolution as Key Characteristics in the Videogame Artifact Design Process.” European Management Journal vol. 24, no. 4(2006): 270-87. doi:10.1016/j.emj.2006.05.003.

Turner, J., L. Thomas, and C. Owen. "Living the Indie Life: Mapping Creative Teams in a 48 Hour Game Jam and Playing with Data.” In Proceedings of The 9th Australasian Conference on Interactive Entertainment: Matters of Life and Death, 1-10. New York, New York, USA: ACM Press, 2013. doi:10.1145/2513002.2513039.

Twidale, M. and D. Nichols. "Collaborative Browsing and Visualisation of the Search Process.” Aslib Proceedings vol. 48, no. 7-8(1996): 177-82. 
Vande M., M. Tomitsch, C. Wimmer, B. Christoph, and T. Grechenig. "Evaluating the Effect of Style in Information Visualization." IEEE Transactions on Visualization and Computer Graphics vol. 18, no. 12(2012): 2739-48. doi:10.1109/TVCG.2012.221.

Vandenbosch, B., S. Fay, and A. Saatçioglu. "Where Ideas Come from: A Systematic View of Inquiry.” Sprouts: Working Papers on Information Systems vol. 1, no. 17(2001): 109-31.

Vazquez, A. “Statistics of Citation Networks.” Trieste, Italy, 2001.

Viegas, F., M. Wattenberg, F. van Ham, J. Kriss, and M. McKeon. "ManyEyes: A Site for Visualization at Internet Scale." IEEE Transactions on Visualization and Computer Graphics vol. 13, no. 6(2007): 1121-28. doi:10.1109/TVCG.2007.70577.

Watts, D. and S. Strogatz. "Collective Dynamics of 'Small-World' Networks.” Nature vol. 393, no. 6684(1998): 440-4422. doi:10.1038/ 30918.

Yamane, S. “Adaptability of the Global Game Jam: A Case Study in Japan.” In Proceedings of the 8th International Conference on the Foundations of Digital Games, 2013.

Zimmerman, J., J. Forlizzi, and S. Evenson. "Research through Design as a Method for Interaction Design Research in HCI.” In Proceedings of the SIGCHI Conference on Human Factors in Computing Systems, 493-503. New York, New York, USA: ACM Press, 2007. doi:10.1145/1240624.1240704.

Zlatic, V., M. Bozicevic, H. Stefancic, and M. Domazet. "Wikipedias: Collaborative Web-Based Encyclopedias as Complex Networks." Physical Review vol. 74, February(2006). doi:10.1103/ PhysRevE.74.016115.

Zook, A. and M. Riedl. “Game Conceptualization and Development Processes in the Global Game Jam.” In Workshop Proceedings of the 
66 ToDIGRA

8th International Conference on the Foundations of Digital Games, 2013. 